\title{
Sclareol prevents ovariectomy-induced bone loss in vivo and inhibits osteoclastogenesis in vitro via suppressing NF-кB and MAPK/ERK signaling pathways
}

Haiming Jin MD, $\mathrm{PhD}^{1,2,3 \#}$, Zhenxuan Shao $\mathrm{MD}^{1,3}$, Qingqing Wang $\mathrm{MD}, \mathrm{PhD}^{1,2,3 \#}$, Jiansen Miao $\mathrm{MD}^{3}$, Xueqing Bai $\mathrm{MD}^{4}$, Jingyu Sun $\mathrm{MD}^{3}$, Qian Liu MD $\mathrm{PhD}^{5}$, Heng Qiu $\mathrm{MD}^{2}$, Chao Wang $\mathrm{MD}^{2}$, Zengjie Zhang $\mathrm{MD}^{1,3}$, Jennifer Tickner $\mathrm{PhD}^{2}$, Xiangyang Wang $\mathrm{MD}, \mathrm{PhD}^{1^{*}}$, Jiake $\mathrm{Xu} \mathrm{MD}, \mathrm{PhD}^{1,2, *}$

${ }^{1}$ Department of Orthopaedic Surgery, The Second Affiliated Hospital and Yuying Children's Hospital of Wenzhou Medical University, Wenzhou, China

2 School of Biomedical Sciences, The University of Western Australia, Perth, Western Australia

${ }^{3}$ The Second School of Medicine, Wenzhou Medical University, Wenzhou, China

${ }^{4}$ Department of Radiology, First Affiliated Hospital of Wenzhou Medical University, Wenzhou, China.

5 Research Centre for Regenerative Medicine and Guangxi Key Laboratory of Regenerative Medicine, Guangxi Medical University, Guangxi, China

\# equally contributed to this work

*corresponding authors

Running title: Sclareol inhibits osteoclastogenesis and prevents bone loss

*Correspondence to: Jiake Xu, School of Biomedical Sciences, The University of Western Australia, Perth, Western Australia, 6009, Australia. Tel: 6189346 2739, E-mail: jiake.xu@uwa.edu.au, Fax: 86-577-88002760

*Correspondence to: Xiangyang Wang, Department of Orthopaedic Surgery, The Second Affiliated Hospital and Yuying Children's Hospital of Wenzhou Medical University, 109 West Xueyuan Road, Wenzhou, Zhejiang Province, 325027, China. Tel: 86-577-88002760, E-mail: xiangyangwang@126.com, Fax: 86-577-88002760 


\begin{abstract}
BACKGROUND/AIMS:

Postmenopausal osteoporosis (PMO) is a progressive disease occurring in elderly postmenopausal women that is characterized by low bone mass and impaired bone quality. Suppressing osteoclastogenesis is considered an effective therapeutic approach for PMO. Sclareol is a natural product (initially isolated from the Salvia sclarea) that possesses immune-regulation and anti-inflammatory effects, but its role in osteoclastic formation and function as well as the PMO remains unknown. In the current study, we aimed to elucidate whether sclareol affects osteoclastogenesis and the progression of PMO.
\end{abstract}

\title{
METHODS:
}

BMMs, that act as osteoclast precursors, were cultured with M-CSF (50 ng/ml) and RANKL (50 ng/ml) for 5 days to generate osteoclasts. Osteoclast differentiation under different treatments was measured by TRAP staining. Osteoclast bone resorption was detected on the hydroxyapatite-coated plate. The expression of osteoclast-related genes were analyzed by RT-PCR and western blotting. The changes of downstream signalling pathways induced by RANKL were confirmed through western blotting and intracellular Ca2+ measurement. Furthermore, an ovariectomy (OVX)-induced bone loss mouse model was established to test the ability of sclareol to prevent bone loss.

\section{RESULTS:}

Sclareol not only inhibited osteoclast formation but also suppressed osteoclast function. The expression of RANKL-induced osteoclast marker gene and protein was also reduced by sclareol treatment. In addition, we found that attenuating the activation of the NF- $\kappa \mathrm{B}$ and MAPK/ERK pathways contributed to the inhibitory effect of sclareol against osteoclastogenesis. Moreover, the in vivo experiment showed that sclareol exerted a protective effect against bone loss in OVX mouse. 
CONCLUSION:

Sclareol suppressed RANKL-induced osteoclast formation and function in vitro by targeting the NF-KB and MAPK/ERK signalling pathways, and prevent the bone loss in OVX mouse. These findings suggest that sclareol has potential value as a therapeutic agent for PMO.

Keywords: Sclareol, Osteoclast, NF-кB, MAPK/ERK, Ovariectomy. 


\section{Introduction}

Bone shaping is the continuous process of coordinated and balanced resorption and formation activities of osteoclasts and osteoblasts[1]. Disorder of the balance between bone formation and bone resorption may lead to metabolic bone diseases, such as postmenopausal osteoporosis (PMO). PMO is a common disease in elderly women and is defined as a skeletal disorder characterized by low bone mass and impaired bone quality, which predisposes a person to an increased risk of fracture[2]. During the progression of PMO, osteoclasts are not kept under control by oestrogen and therefore become overactive, which leads to the temporary uncoupling of bone resorption and formation, resulting in an overall net bone loss[3]. Therefore, agents that inhibit the formation and function of osteoclasts and thus can be a promising therapeutic target for PMO are sought.

Osteoclasts are multinucleate giant cells derived from haematopoietic precursor cells of monocyte/macrophage lineage. The formation and differentiation of osteoclasts are regulated by osteoblasts through the synthesis of macrophage colony-stimulating factor (M-CSF) and receptor activator of nuclear factor (NF)- $\kappa$ B ligand (RANKL)[4]. M-CSF promotes the proliferation and survival of osteoclast precursor cells and upregulates the expression of RANK[5]. RANKL binds to its receptor RANK, and sequentially recruits TNF receptor-associated factor 6 (TRAF6) in the osteoclast precursor cells. TRAF6 activates downstream signalling pathways, including NF- $\kappa \mathrm{B}$ and mitogen-activated protein kinases (MAPKs) $[6,7]$. Ultimately, the activation of NF- $\mathrm{kB}$ and MAPKs contribute to the expression of transcription factors such as c-Fos and nuclear factor of activated T cells, cytoplasmic 1 (NFATc1), which are crucial for the differentiation of osteoclast precursors[8, 9]. Thus, blocking these crucial signalling pathways induced by RANKL is a potential therapeutic target for treating PMO.

Sclareol, a phytochemical labdane diterpene isolated form Salvia sclarea, has been reported to possess a series of functions, such as immune-regulatory[10], 
anti-inflammatory[11] and anticancer[12] effects. However, the influence of sclareol on osteoclastogenesis still remains unknown. Recently, sclareol was found to ameliorate lipopolysaccharide-induced pulmonary inflammation through the

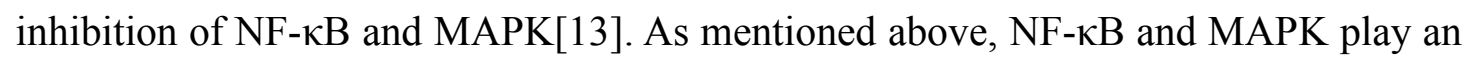
indispensable role in the formation and differentiation of osteoclasts. Therefore, in our study, we investigated the inhibitory effect of sclareol on RANKL-induced osteoclastic formation and function as well as the underlying mechanism. Furthermore, an ovariectomy (OVX)-induced bone loss mouse model was established to mimic PMO, and the protective role of sclareol against bone loss from PMO was examined.

\section{Materials and methods}

\section{Ethics Statement}

The study was approved by the Animal Care and Use Committee at the Wenzhou Medical College. All surgical interventions, treatments, and post-operative animal care procedures were performed in accordance with the National Institutes of Health (NIH) Guide for the Care and Use of Laboratory Animals.

\section{Reagents}

Sclareol (purity $>98 \%$ ) was purchased from Nantong Feiyu Biological Technology Co, Ltd. (Nantong, China). Sclareol was dissolved in DMSO as a $100 \mathrm{mM}$ stock solution and stored at $-20{ }^{\circ} \mathrm{C}$. Further dilution was performed in cell culture medium. The primary antibody of NFATc1, c-Fos, Integrin- $\beta 3$, CTSK, V-ATPase $\mathrm{d} 2$ and $\beta$-actin were obtained from Santa Cruz Biotechnology (San Jose, CA). IкB- $\alpha$, ERK/p-ERK and P38/p-P38 antibodies were purchased from Cell Signaling Technologies (Beverly, MA, USA). The MTS assay kit and luciferase assay system were purchased from Promega (Madison, WI, USA). A Leukocyte acid phosphatase staining kit was purchased from Sigma-Aldrich (Sydney, Australia). Recombinant macrophage colony stimulating factor (M-CSF) was obtained from R\&D Systems (Minneapolis, MN). Recombinant GST-rRANKL protein was synthesized and purified by Jiake $\mathrm{Xu}$ as 
previously describe[14]. The cell culture medium, alpha modified minimal essential medium ( $\alpha$-MEM) and fetal bovine serum (FBS), were purchased from Thermo Fisher Scientific (Scoresby, Vic, Australia).

\section{Cell Culture}

Bone marrow macrophages (BMMs) was flushed from tibiae and femurs, which were isolated from 6-week-old C57/BL6 mice. Then BMMs were cultured for 3 days in a-MEM supplemented with $10 \%$ fetal bovine serum (FBS), $1 \%$ antibiotic mixture of penicillin, streptomycin and $50 \mathrm{ng} / \mathrm{mL}$ M-CSF, and incubated at $37^{\circ} \mathrm{C}$ and humidified at $5 \% \mathrm{CO} 2$. Culture medium was changed every 2 days, and cells were passaged when $90-100 \%$ confluence had been attained.

\section{TRAP staining}

BMMs were seeded into 96-well plates at a density of $5 \times 10^{3}$ cells per well and allowed to adhere overnight. Following, RANKL $(50 \mathrm{ng} / \mathrm{ml})$ were used for osteoclast induction, which was treated in the presence or absence of different concentrations of sclareol. The medium was replaced every two days. After 5 days, cells were fixed with 4\% glutaraldehyde and TRAP staining assay was then performed using a leukocyte acid phosphatase kit (Sigma) according to the manufacturer's instructions. The number of TRAP-positive multinucleated cells (nuclei $>3$ ) were counted under the microscope (Nikon Corporation, Tokyo, Japan).

\section{Cytotoxicity assays}

The passage 2 BMMs were planted in 96 -well plates $\left(5 \times 10^{3}\right.$ cells/well $)$ and incubated in $\alpha$-MEM with $10 \% \mathrm{FBS}$ and $50 \mathrm{ng} / \mathrm{mL} \mathrm{M}-\mathrm{CSF}$ at $37^{\circ} \mathrm{C}$ for $24 \mathrm{~h}$. Then, the cells were treated without or with different concentration $(0,1,2.5,5$ and $10 \mathrm{um})$ of sclareol for 48 hours. After 48 hours, MTS solution $(20 \mu \mathrm{L} /$ well $)$ was added and incubated with cells for $2 \mathrm{~h}$. The absorbance at $490 \mathrm{~nm}$ was read with a microplate reader (ThermoFisher, Waltham, MA, USA)

\section{Resorption pit assay}


To measure the influence of sclareol on osteoclasts function, BMMs were seeded into 6-well plate with $50 \mathrm{ng} / \mathrm{mL}$ RANKL and $50 \mathrm{ng} / \mathrm{mL}$ M-CSF until mature oeteoclasts were generated. Then mature osteocalsts were gently detached from the wells using cell dissociation solution (Sigma, St. Louis, MO, USA), and cultured into hydroxyapatite coated 96-well plates containing RANKL (50 ng/mL), M-CSF(50 $\mathrm{ng} / \mathrm{mL}$ ) and different concentrations of sclareol. After 48 hours, resorption areas were captured using a standard light microscopy and quantified using Image J software (NIH, Bethesda, MD, USA).

\section{Fibrous Actin Ring Observation}

BMMs were seeded into a 96-well plate at a density of $5 \times 10^{3}$ cells per well and incubated in $\alpha$-MEM with $10 \%$ FBS, $50 \mathrm{ng} / \mathrm{mL} \mathrm{M}-\mathrm{CSF}, 50 \mathrm{ng} / \mathrm{mL}$ RANKL and the indicated concentration of sclareol for 5 days. The medium was replaced every 2 days. After the treatment period, cells were fixed with paraformaldehyde (4\%) for $15 \mathrm{~min}$ at room temperature. After washing with FBS three times, cells were permeabilized with $0.25 \%$ Triton X-100 and blocked with $3 \%$ BSA in PBS. Next, the F-actin rings were stained with rhodamine conjugated phalloidin (Eugene, OR, USA), while the cell nuclei were stained with DAPI. Then images were collected using confocal laser scanning microscopy (Nikon, Tokyo, Japan)

\section{RNA Isolation And Quantitative RT-PCR Analysis}

Quantitative real-time polymerase chain reaction (qRT-PCR) was performed to analyze specific gene expression during osteoclastogenesis, such as TRAcP, MMP9, CTSK and V-ATPase d2. Briefly, BMMs were seeded at $8 \times 10^{4} /$ well in 6-well plates. The cells were cultured with $50 \mathrm{ng} / \mathrm{ml} \mathrm{M}-\mathrm{CSF}$ and $50 \mathrm{ng} / \mathrm{ml}$ RANKL, with or without $10 \mu \mathrm{M}$ sclareol for $0,1,3$, or 5 days. Total RNA was isolated from cell cultures using Trizol reagent according to the manufacturer's instructions (ThermoFisher Scientific, Scoresby, Australia). The complementary DNA (cDNA) was synthesized using $1 \mu \mathrm{g}$ of RNA template. Then cDNA was amplified using a PCR Premix Kit (Invitrogen, Carlsbad, CA, USA). The cycling parameters using for RT-PCR reactions were $94{ }^{\circ} \mathrm{C}$ 
for $40 \mathrm{~s}, 60{ }^{\circ} \mathrm{C}$ for $40 \mathrm{~s}, 72^{\circ} \mathrm{C}$ for $40 \mathrm{~s}, 72^{\circ} \mathrm{C}$ for $5 \mathrm{~min}$, and were performed for 40 cycles. Relative levels of tested genes were normalized to that of HPRT. Primers for real-time PCR are listed in Table 1.

\section{Luciferase Reporter Assays}

The effects of sclareol on RANKL-induced NF-kB and NFATc1 activation were measured using RAW264.7 cells. RAW264.7 cells were stably transfected with either an NF-кB-responsive luciferase construct or an NFATc1-responsive luciferase reporter construct. Briefly, BMMs were seeded in 48 -well plates at a density of $5 \times 10^{3}$ cells/well overnight. The next day, cells were pretreated with different concentrations of sclareol for $1 \mathrm{~h}$. Following, pre-treatment cells were subsequently stimulated with RANKL (50 ng/mL) ( $6 \mathrm{~h}$ for NF- $\mathrm{kB}$ and $24 \mathrm{~h}$ for NFATc1) in the presence of sclareol. Luciferase activity was measured using the luciferase reporter assay system according to the manufacturer's protocol (Promega, Sydney, Australia).

\section{Western Blotting}

Western blotting was performed using routine protocols. The protein was extracted from treated cells using RIPA buffer. Cellular proteins were resolved by $8-12 \%$ sodium dodecyl sulfate-polyacrylamide gel electrophoresis (SDS-PAGE) and transferred to a polyvinylidene fluoride (PVDF) membranes (GE Healthcare, Silverwater, Australia). Then membrane was blocked with 5\% skim milk for $2 \mathrm{~h}$ at room temperature and incubated overnight at $4{ }^{\circ} \mathrm{C}$ with the primary antibody. After washed in TBST for three times, the membrane was incubated with an secondary antibody. Protein bands were then visualized using an enhanced chemiluminescence (ECL) system (Amersham Pharmacia Biotech, Sydney, Australia).

\section{Intracellular $\mathrm{Ca}^{2+}$ Measurement}

Intracellular $\mathrm{Ca}^{2+}$ measurement was performed to detect the activation of calcium signalling pathway induced by RANKL. Briefly, BMMs were seeded into 48-well plates with M-CSF $(50 \mathrm{ng} / \mathrm{mL})$ at a density of $1 \times 10^{4}$ cells per well for $24 \mathrm{~h}$; next, the 
cells were treated with or without sclareol $(10 \mu \mathrm{M})$ and RANKL $(50 \mathrm{ng} / \mathrm{mL})$ in the presence of M-CSF $(50 \mathrm{ng} / \mathrm{mL})$ for another $24 \mathrm{~h}$. Then, the cells were washed twice with assay buffer (Hanks' balanced salt solution supplemented with $1 \mathrm{mM}$ probenecid and 1\% FBS) and stained with Fluo4 staining solution (Fluo4-AM dissolved in 20\% Pluronic F127 (w/v) in DMSO diluted in assay buffer) in the dark at $37^{\circ} \mathrm{C}$ for $45 \mathrm{~min}$. Next, the intracellular free calcium was detected by an inverted fluorescence microscope (Nikon Ti-U) at an excitation wavelength of $488 \mathrm{~nm}$. To obtain the changes of $\mathrm{Ca}^{2+}$ oscillation, images of different group were captured every 2 sec for 3 min, and the results were recorded and analysed by Nikon Basic Research Software (cells with two or more oscillating peaks were included). The average amplitude of each oscillating cell under different treatments was analysed.

\section{Animal Experiments}

Seven-week-old female C57BL/6 mice were purchased from the Animal Center of Chinese Academy of Sciences, Shanghai, China, and randomly divided into sham group $(n=10)$, OVX group $(n=10)$, and sclareol group $(n=10)$. The mice were kept for at least 7 days before bilateral ovariectomy or sham operation. The bilateral OVX procedures were performed in OVX group and sclareol group using dorsal approach, and the rat in sham group were subjected to the same surgical procedure with the ovary exposed for $1 \mathrm{~min}$, but did not undergo the ovariectomy. After the surgery, the animals were allowed to recover from surgery for one week prior to the experiments. Sclareol group received sclareol $(10 \mathrm{mg} / \mathrm{kg} /$ day $)$ dissolved in CMC by intragastric administration on a daily basis until the mice were sacrificed. Meanwhile, mice in sham group and OVX group were administered an equivalent volume of CMC. All mice were humanely euthanized after 8 -weeks following treatment, and their femur bone samples were collected. The bone loss and microstructure of the distal femur were observed using micro-CT scanning.

\section{Micro-CT Scanning}

The micro-architecture on the trabecular bone of distal femur was determined using a 
high-resolution micro-CT (Skyscan 1176; Bruker, Belgium). Briefly, the trabecular parameters were evaluated by micro-CT Skyscan CTAn software, including the bone volume per tissue volume (BV/TV), trabecular number (Tb.N), trabecular thickness (Tb.Th), and trabecular separation (Tb.Sp). The three-dimensional (3D) bone structure images were reconstructed by the Mimics 18.0 software (Materialise, Leuven, Belgium).

\section{Statistical Analysis}

All experiments were performed independently for at least three times. All results were expressed as the mean \pm standard error of the mean (SEM). Intergroup comparisons were made using one-way ANOVA, followed by the Tukey's test. Probability values of $\mathrm{P}<0.05$ were considered statistically significant.

\section{Results}

\section{Sclareol suppressed RANKL-induced osteoclastogenesis in vitro}

To investigate the inhibitory effect of sclareol on osteoclast formation, BMMs were treated with various concentrations $(0,1,2.5,5$ and $10 \mu \mathrm{M})$ of sclareol in the presence of M-CSF and RANKL. As shown in Figure 1A and Figure 1C, sclareol exerted a dose-dependent inhibition on RANKL-induced osteoclastogenesis compared to the RANKL-induced group, which is measured by counting TRACP positive multinucleated cells $(>=3)$. Furthermore, in order to clarify at what stage sclareol play the most important role, the BMMs were treated with sclareol during different periods (1-3 days, 3-5 days, 5-6 days). We found the process of osteoclastogenesis was more suppressed while treated with sclareol during 1-3 days, and the inhibitory effect decreased during 3-5 and 5-6 days (Fig. 1B, C). These results indicated that sclareol dose-dependently inhibited RANKL-induced osteoclastogenesis and mainly acted at the early stage during osteoclastogenesis. Then, cell viability assays was performed to evaluate the toxicity of sclareol on osteoclastogenesis. As indicated in Figure 1D, sclareol has no obvious toxicity on osteoclastogenesis at the concentration up to 10 $\mu \mathrm{M}$. Therefore, the inhibitory effect of sclareol on osteoclastogenesis is not due to the 
cytotoxic effect.

\section{Sclareol inhibits RANKL-induced F-actin ring formation in osteoclasts}

To further evaluate the inhibitory effect of sclareol on osteoclastogenesis, we examined how sclareol affected RANKL-induced osteoclast actin ring formation, which is a prerequisite for osteoclast bone resorption and is the most obvious characteristic of mature osteoclasts during osteoclastogenesis[15]. The F-actin rings were stained with rhodamine conjugated phalloidin, and the cell nuclei were stained with DAPI. The result indicated that BMMs treated with higher concentrations of sclareol grew into smaller osteoclasts with fewer nuclei (Fig. 2). The results showed that sclareol attenuated RANKL-induced F-actin ring formation in osteoclasts.

\section{Sclareol attenuates RANKL-induced osteoclast bone resorption}

To determine the effect of sclareol on osteoclast function, BMMs were cultured in $\alpha$-MEM with RANKL and M-CSF until mature osteoclasts formed. Then cells were seeded into hydroxyapatite coated plates for bone resorption pit assay. A decrease in bone resorption was observed with an increase in sclareol concentration (Fig. 3A, C). After analysing the results, the area resorbed per OC also diminished in a dose-dependent manner (Fig. 3E). Thus, these data indicated that sclareol inhibited osteoclast bone resorption.

\section{Sclareol down-regulates osteoclast relative gene expressions}

Osteoclast differentiation is regulated directly by the expression of a series of related marker genes, such as CTSK, NFATc1, TRAcP and calcitonin receptor (CTR)[16, 17]. Then qRT-PCR was performed to clarify the expression of relative maker genes in osteoclasts which were treated with or without various concentrations of sclareol. As shown in Figure 4, the mRNA expressions of CTSK, TRAcP, NFATc1 and CTR were significantly down-regulated by sclareol in a dose-dependent manner.

Sclareol inhibits RANKL-induced NFATc1 activation and downstream protein expression in BMMs 
It is well-known that NFATc1 is a key transcription factors during RANKL-induced osteoclastogenesis, which also is a master switch for regulating terminal differentiation of osteoclasts[17]. Luciferase reporter assay was performed to confirm the effect of sclareol on the expression of NFATc1. The results revealed that the NFATc1 luciferase activity was suppressed by the treatment of sclareol, especially at concentration of $10 \mu \mathrm{M}$ (Fig. 5A). In addition, we performed western blotting to measure the expression level of NFATc1 and NFATc1 downstream protein. As shown in Figure 5B, the up-regulation protein level of NFATc1 induced by RANKL was reduced by sclareol treatment during the osteoclast differentiation. Similarily, the expression of NFATc1 downstream protein including c-Fos, and V-ATPase-d2 was inhibited on day 3 and 5, while the expression of CTSK, and integrin $\beta$ was inhibited on day 5.

\section{Sclareol inhibits osteoclastogenesis by suppressing NF-אB and MAPK/ERK pathways}

The differentiation of osteoclast precursors into mature osteoclasts is associated with RANKL-stimulated NF- $\mathrm{KB}$ activation, which play a crucial role in osteoclastogenesis. Western blotting and luciferase reporter assay were conducted to detect the effect of sclareol on NF- $\kappa$ B signaling pathway. The results showed that NF- $\kappa B$ luciferase activity was significantly suppressed by sclareol in a dose-dependent manner, especially at the concentration of 5 and $10 \mu \mathrm{M}$ (Fig. 6A). Furthermore, according to western blotting result, sclareol significantly attenuated RANKL-induced degradation of $I \kappa B \alpha$, which is an inhibitory subunit of NF- $\kappa B$ and serves as a key factor in NF- $\kappa B$ signaling pathway (Fig. 6B). Besides NF- $\mathrm{B}$, RANKL also stimulated MAPK signaling pathway during osteoclast differentiation. As shown in Figure 6C, consistent with previous reports, RANKL stimulated the phosphorylation of ERK and P38 within 20 min (Fig. 4). However, the treatment of sclareol obviously suppressed the phosphorylation of ERK while have no obvious inhibitory effect on the phosphorylation of P38. Furthermore, we performed intracellular $\mathrm{Ca}^{2+}$ measurement 
to evaluate the effect of sclareol on calcium signalling pathway. The results indicated that sclareol failed to alter $\mathrm{Ca}^{2+}$ oscillation induced by RANKL (Fig. S1). Overall, sclareol inhibited osteoclastogenesis mainly by suppressing NF- $\kappa \mathrm{B}$ and MAPK/ERK pathways.

\section{Sclareol prevents OVX-induced bone loss in vivo}

To examine whether sclareol prevents OVX-induced bone loss, we used the OVX mouse model to mimic menopause-induced bone loss in women. A significant decline of bone volume and trabecular number with increased trabecular space was observed in the OVX group compared to the sham group by micro-CT scanning and 3D reconstruction (Fig. 7A). However, sclareol administration reversed all this alterations induced by OVX, with markedly increases in BV/TV and Tb.N, and a decrease in Tb.Sp (Fig. 7B-D). As with most researches, the value of Tb.Th kept unchanged in all groups (Fig. 7E).

\section{Discussion}

PMO, characterized by low bone mass and micro-architectural alterations, is a common disease in women after menopause that results in bone fragility and an increased risk of fractures. PMO is due to progressive and excessive bone resorption by osteoclasts, which is attributable to the dearth of oestrogen[3]. Thus, the search for drugs suppressing the formation and function of osteoclasts can be a valuable treatment for this pathological bone loss. Numerous pharmacological agents and therapies are clinically applied for the treatment of PMO, but with serious adverse effects[18-20]. For example, hormone replacement therapy (HRT) is a treatment for PMO that enhances the level of oestrogen and thus reduces bone loss. Nevertheless, long-term HRT has been found to increase the risk of heart attack, stroke, and breast and uterine cancer[21]. In recent years, TCMs have garnered increasing attention for their efficacy against various diseases with low side effects[22, 23]. Sclareol, mainly isolated and purified form the leaves and flowers of Salvia Sclarea, has been reported to exert many effects on anti-tumour, anti-inflammatory and immune-regulatory 
activity[10-12]. However, sclareol's influence on osteoclastogenesis still remains unknown. In this study, for the first time, we demonstrated that sclareol inhibited RANKL-induced osteoclast formation and function and had a protective effect against bone loss in OVX mice.

Osteoclasts, which serve as the only bone-resorptive cells in the body, play a dispensable role in the balance between bone formation and bone resorption. The differentiation of osteoclasts mainly depends on the presence of two key factors, macrophage colony stimulation factor (M-CSF), and receptor activator of NF-B ligand (RANKL). M-CSF plays a crucial role in osteoclast precursor cell proliferation, whereas RANKL induces the differentiation of osteoclast precursor into osteoclasts[24]. In our study, we found that sclareol had little effect on M-CSF-induced osteoclast precursor cell proliferation by an MTS assay, while sclareol dose-dependently inhibited RANKL-induced osteoclastic formation and function.

The binding of RANKL to its receptor RANK leads to the recruitment of TRAF6 and the subsequent activation of a broad range of downstream signalling pathways, including NF-kB, MAPKs and calcium[7, 25, 26]. It is well established that the activation of NF- $\kappa \mathrm{B}$ is involved in the phosphorylation of $\mathrm{I} \kappa \mathrm{B} \alpha$ and the $\mathrm{p} 65$ subunit as well as the subsequent degradation of $\mathrm{I} \kappa \mathrm{B} \alpha$. Once liberated from $\mathrm{I} \kappa \mathrm{B} \alpha$, the NF- $\mathrm{B}$ p65 subunit translocates from the cytoplasm to the nucleus to conduct transcriptional activity[27]. Our data revealed that sclareol not only inhibited the degradation of $\mathrm{I} \kappa \mathrm{B} \alpha$ protein but also suppressed the transcriptional activity of NF- $\kappa \mathrm{B}$. In addition to the NF- $\kappa \mathrm{B}$ signalling pathway, activation of the MAPK also plays a crucial role in osteoclastogenesis[5,28]. The extracellular-signal-regulated kinase (ERK) pathway is one of the major signalling MAPK signalling pathways, which plays an important role in the survival, apoptosis and differentiation of osteoclasts. The inhibition of ERK protein kinase reportedly attenuates RANKL-induced osteoclastogenesis[9, 29, 30]. In our study, we found that sclareol significantly suppressed the phosphorylation of ERK 
but had no effect on P38. Calcium signalling pathway also was reported to play an important role in the osteoclastogenesis[26]. RANKL-induced $\mathrm{Ca}^{2+}$ oscillation could lead the expression of transcription factors in osteoclast differentiation. However, the results of intracellular $\mathrm{Ca}^{2+}$ Measurement indicated that sclareol had no significant effect on $\mathrm{Ca}^{2+}$ oscillation. Taken together, these data indicated that inhibiting or delaying the activation of the NF- $\mathrm{BB}$ and MAPK/ERK pathways contributed to the inhibitory effect of sclareol against osteoclastogenesis (Fig. 8).

Activation of the NF- $\mathrm{KB}$ and MAPK signalling pathways promotes the expression of several transcription factors such as c-Fos, activator protein 1 (AP1) and NFATc1[31-33]. NFATc1 is the most strongly induced transcription factor gene by RANKL stimulation and serves as a 'master regulator' to drive the expression of osteoclast-specific genes such as TRAP, CTR, Cathepsin K, and V-ATPase-d2, most of which also participate in the process of osteoclastic bone resorption[34]. In the present study, we found that the RANKL-induced upregulation of c-Fos, CTSK, V-ATPase-d2, NFATc1 and Integrin $\beta 3$ was inhibited by sclareol at the protein level. The mRNA levels of TRAcP, NFATc1, CTSK and the CTR were also reduced by sclareol in a dose-dependent manner.

In this study, an ovariectomy-induced osteoporosis mouse model was examined for further investigation of the therapeutic effect of sclareol in vivo. By using micro-CT scanning and $3 \mathrm{D}$ reconstruction, we found that the bone loss and micro-architecture change induced by OVX were markedly reversed by sclareol administration. Taken together, the in vivo and in vitro experiments suggested that sclareol possesses potential therapeutic value in preventing the progression of PMO.

In conclusion, for the first time, our findings demonstrate that sclareol suppressed RANKL-induced osteoclast formation and function in vitro by targeting the NF-KB and MAPK/ERK signalling pathways. Moreover, the oral administration of sclareol was effective in suppressing bone loss in OVX mice. These results suggest that 
sclareol could be a potential agent for the treatment of osteoclast-related diseases, such as PMO.

\section{Acknowledgments}

This work is supported by grant from Western Australia Medical \& Health Research Infrastructure Fund, Arthritis Australia foundation, The University of Western Australia (UWA) Research Collaboration Awards, and the National Health and Medical Research Council (NHMRC, No.1107828, 1027932). It is also funded in part by National Natural Science Foundation of China (No. 81871806), Zhejiang Public service technology research program / social development (No. LGF18H060008) and the Guangxi Natural Science Foundation (No. 2015GXNSFDA139019). Drs. Haiming Jin, Qingqing Wang and Jiake Xu made mutual collaborative visits.

\section{Conflict of interest}

The authors declare no conflict of interest. 
Table. 1 Primer sequences used in qRT-PCR

\begin{tabular}{|c|c|c|}
\hline Gene & Forward primer & Reverse primer \\
\hline TRAcP & 5'-TGTGGCCATCTTTATGCT -3' & 5'-GTCATTTCTTTGGGGCTT -3' \\
\hline NFATc1 & 5'-CAACGCCCTGACCACCGATAG-3' & 5'-GGCTGCCTTCCGTCTCATAGT -3' \\
\hline CTSK & 5'-GGGAGAAAAACCTGAAGC-3' & 5'-ATTCTGGGGACTCAGAGC-3' \\
\hline $\begin{array}{c}\text { Calcitonin } \\
\text { receptor }\end{array}$ & 5'-TGGTTGAGGTTGTGCCCA-3' & 5'-CTCGTGGGTTTGCCTCATC-3' \\
\hline HPRT & 5'-GTTGGGCTTACCTCACTGCT-3' & 5'-TAATCACGACGCTGGGACTG-3' \\
\hline
\end{tabular}

\section{Figure Legends}

Figure 1. Sclareol suppressed RANKL-induced osteoclastogenesis in vitro. (A) Representative TRAcP staining images of RANKL-induced osteoclast formation treated with different concentrations $(1,2.5,5$, and $10 \mu \mathrm{M})$ of sclareol for 5 days as well as with $10 \mu \mathrm{M}$ sclareol on indicated days. Scale bar, $400 \mu \mathrm{m}$. Scale bar in enlarged images, $100 \mu \mathrm{m}$. (B) The schematic diagram of $10 \mu \mathrm{M}$ sclareol treatment time in A. (C) Quantification of $\mathrm{TRAP}^{+}$multinucleated cells (nuclei>3) shown in A. (D) Cell viability under different concentrations of sclareol was evaluated by MTS assay. Data are presented as mean $\pm \mathrm{SEM}, * \mathrm{P}<0.05, * * \mathrm{P}<0.01$ relative to the control group. ${ }^{\#} \mathrm{P}<0.05,{ }^{\#} \mathrm{P}<0.01$ relative to the $\mathrm{RANKL}$-induced group. $\mathrm{n}=3$.

Figure 2. Sclareol inhibits RANKL-induced F-actin ring formation in osteoclasts. (A) Cells after the treatment were fixed and stained with rhodamine (red) for F-actin rings, and Hoechst (blue) for nuclei. Scale bar, $200 \mu \mathrm{m}$. (B) Quantification of the osteoclasts (nuclei $\geqslant 3$ ) in each field. (C) Quantification of the average nucleus number in each group. Data are presented as mean $\pm \mathrm{SEM}, * \mathrm{P}<0.05, * * \mathrm{P}<0.01$ relative to the control group. $\# \mathrm{P}<0.05, \# \# \mathrm{P}<0.01$ relative to the $\mathrm{RANKL}$-induced group. $\mathrm{n}=3$.

Figure 3. Sclareol attenuates RANKL-induced osteoclast bone resorption. (A,B) Representative images of resorbing pits and TRAcP stained osteoclasts on hydroxyapatite-coated plate. The white areas are where the osteoclasts have resorbed the hydroxyapatite-coated plate. Scale bar, $400 \mu \mathrm{m}$. Scale bar in enlarged images, 100 $\mu \mathrm{m}$. (C,D) The osteoclasts number per well, resorbed areas in total of each well and 
per cell were quantified using ImageJ software. Data are presented as mean $\pm \mathrm{SEM}$, $* \mathrm{P}<0.05, * * \mathrm{P}<0.01$ relative to the control group. $\mathrm{n}=3$.

Figure 4. Sclareol down-regulates osteoclast relative gene expressions. (A-D) Expression of mRNA for RANKL-induced osteoclast marker genes, TRAP, NFATc1, Ctsk, and calcitonin receptor, at day 5 of different groups were examined by qRT-PCR analyses. HPRT were used as loading controls. Data are presented as mean $\pm \mathrm{SEM}$, ${ }^{*} \mathrm{P}<0.05,{ }^{*} * \mathrm{P}<0.01$ relative to the control group. $\# \mathrm{P}<0.05, \# \# \mathrm{P}<0.01$ relative to the RANKL-induced group. $\mathrm{n}=3$.

Figure 5. Sclareol inhibits RANKL-induced NFATc1 activation and downstream protein expression in BMMs. (A) The effect of sclareol on RANKL-induced transcriptional activity of NFATc1 were examined using a luciferase reporter gene assay. (B) Expression levels of the RANKL-induced osteoclast marker proteins, NFATc1, c-Fos, CTSK, V-ATPase d2, and Integrin- $\beta 3$, were examined by western blotting analyses on the indicated days. Data are presented as mean $\pm \mathrm{SEM},{ }^{*} \mathrm{P}<0.05$, $* * \mathrm{P}<0.01$ relative to the control group. $\# \mathrm{P}<0.05, \# \# \mathrm{P}<0.01$ relative to the RANKL-induced group. $\mathrm{n}=3$.

Figure 6. Sclareol inhibits osteoclastogenesis by suppressing NF- $\mathrm{B}$ and MAPK/ERK pathways. (A) The effect of sclareol on RANKL-induced transcriptional activity of $\mathrm{NF}-\kappa \mathrm{B}$ were examined using a luciferase reporter gene assay. (B-D) Representative images of western blotting and quantitative analysis demonstrating the effect of sclareol on I $\mathrm{B} \alpha$ degradation, phosphorylation of ERK1/2, and phosphorylation of P38 during RANKL-induced osteoclastogenesis time course. Data are presented as mean $\pm \mathrm{SEM},{ }^{*} \mathrm{P}<0.05,{ }^{*} * \mathrm{P}<0.01$ relative to the control group, $\# \mathrm{P}<0.05, \# \# \mathrm{P}<0.01$ relative to the RANKL-induced group. \&P $<0.05, \& \& \mathrm{P}<0.01$ relative to the RANKL-induced group on the same indicated time. $\mathrm{n}=3$.

Figure 7. Sclareol prevents OVX-induced bone loss in vivo. (A) Representative 
reconstructed trabecular and cortical bone structure images of distal femoral bone in different group. (B-E) Quantitative analysis of distal femoral bone volume per tissue volume (BV/TV), trabecular number (Tb.N), trabecular thickness (Tb.Th), and trabecular separation (Tb.Sp) in each group. Data are presented as mean $\pm \mathrm{SEM}$, ${ }^{*} \mathrm{P}<0.05, * * \mathrm{P}<0.01$ relative to the sham group. $\# \mathrm{P}<0.05, \# \# \mathrm{P}<0.01$ relative to the OVX group, $\mathrm{n}=6$.

Figure 8. Schematic model of the signalling mechanisms underlying the inhibitory effect of sclareol on RANKL-induced osteoclastogenesis.

Figure S1. Sclareol has little effect on $\mathrm{Ca}^{2+}$ oscillation induced by RANKL. (A-C) Representative images of $\mathrm{Ca}^{2+}$ oscillation under the different treatments. (D) Quantitative analysis showing that sclareol had no significant effect on RANKL-induced Ca2+ oscillation.

\section{Reference}

1 Masi L, Brandi ML: Physiopathological basis of bone turnover. The quarterly journal of nuclear medicine : official publication of the Italian Association of Nuclear Medicine (AIMN) [and] the International Association of Radiopharmacology (IAR) 2001;45:2-6.

2 Lagari VS, Levis S: Phytoestrogens in the prevention of postmenopausal bone loss. Journal of clinical densitometry : the official journal of the International Society for Clinical Densitometry 2013;16:445-449.

3 D'Amelio P, Grimaldi A, Di Bella S, Brianza SZM, Cristofaro MA, Tamone C, Giribaldi G, Ulliers D, Pescarmona GP, Isaia G: Estrogen deficiency increases osteoclastogenesis up-regulating T cells activity: a key mechanism in osteoporosis. Bone 2008;43:92-100.

4 Asagiri $\mathrm{M}$, Takayanagi $\mathrm{H}$ : The molecular understanding of osteoclast differentiation. Bone 2007;40:251-264.

5 Boyle WJ, Simonet WS, Lacey DL: Osteoclast differentiation and activation. Nature 2003;423:337-342.

6 Takayanagi H: Mechanistic insight into osteoclast differentiation in osteoimmunology. Journal of molecular medicine (Berlin, Germany) 2005;83:170-179.

7 Leibbrandt A, Penninger JM: RANK/RANKL: regulators of immune responses and bone physiology. Annals of the New York Academy of Sciences 2008;1143:123-150. 
8 Miyazaki T, Katagiri H, Kanegae Y, Takayanagi H, Sawada Y, Yamamoto A, Pando MP, Asano T, Verma IM, Oda H, Nakamura K, Tanaka S: Reciprocal role of ERK and NF-kappaB pathways in survival and activation of osteoclasts. The Journal of cell biology 2000;148:333-342.

9 Monje P, Hernandez-Losa J, Lyons RJ, Castellone MD, Gutkind JS: Regulation of the transcriptional activity of c-Fos by ERK. A novel role for the prolyl isomerase PIN1. The Journal of biological chemistry 2005;280:35081-35084.

10 Noori S, Hassan ZM, Mohammadi M, Habibi Z, Sohrabi N, Bayanolhagh S: Sclareol modulates the Treg intra-tumoral infiltrated cell and inhibits tumor growth in vivo. Cellular immunology 2010;263:148-153.

11 Huang GJ, Pan $\mathrm{CH}$, Wu $\mathrm{CH}$ : Sclareol exhibits anti-inflammatory activity in both lipopolysaccharide-stimulated macrophages and the lambda-carrageenan-induced paw edema model. Journal of natural products 2012;75:54-59.

12 Dimas K, Papadaki M, Tsimplouli C, Hatziantoniou S, Alevizopoulos K, Pantazis P, Demetzos C: Labd-14-ene-8,13-diol (sclareol) induces cell cycle arrest and apoptosis in human breast cancer cells and enhances the activity of anticancer drugs. Biomedicine \& pharmacotherapy $=$ Biomedecine $\&$ pharmacotherapie 2006;60:127-133.

13 Hsieh YH, Deng JS, Pan HP, Liao JC, Huang SS, Huang GJ: Sclareol ameliorate lipopolysaccharide-induced acute lung injury through inhibition of MAPK and induction of HO-1 signaling. International immunopharmacology 2017;44:16-25.

14 Xu J, Tan JW, Huang L, Gao XH, Laird R, Liu D, Wysocki S, Zheng MH: Cloning, sequencing, and functional characterization of the rat homologue of receptor activator of NF-kappaB ligand. Journal of bone and mineral research : the official journal of the American Society for Bone and Mineral Research 2000;15:2178-2186.

15 Jurdic P, Saltel F, Chabadel A, Destaing O: Podosome and sealing zone: specificity of the osteoclast model. European journal of cell biology 2006;85:195-202.

16 !!! INVALID CITATION !!!

17 Zhao Q, Shao J, Chen W, Li YP: Osteoclast differentiation and gene regulation. Frontiers in bioscience : a journal and virtual library 2007;12:2519-2529.

18 Hamdy RC, Chesnut CH, 3rd, Gass ML, Holick MF, Leib ES, Lewiecki ME, Maricic M, Watts NB: Review of treatment modalities for postmenopausal osteoporosis. Southern medical journal 2005;98:1000-1014; quiz 1015-1007, 1048.

19 Rizzoli R, Boonen S, Brandi ML, Burlet N, Delmas P, Reginster JY: The role of calcium and vitamin D in the management of osteoporosis. Bone 2008;42:246-249.

20 Mavrokokki T, Cheng A, Stein B, Goss A: Nature and frequency of bisphosphonate-associated osteonecrosis of the jaws in Australia. Journal of oral and maxillofacial surgery : official journal of the American Association of Oral and Maxillofacial Surgeons 2007;65:415-423.

21 Calleja-Agius J, Brincat MP: Hormone replacement therapy post Women's Health Initiative study: where do we stand? Current opinion in obstetrics \& gynecology 2008;20:513-518.

22 Yang Y, Li Y: Systematic Investigation of Ginkgo Biloba Leaves for Treating Cardio-cerebrovascular Diseases in an Animal Model. 2017;12:1363-1372.

23 Wong WC, Lee A, Wong SY, Wu SC, Robinson N: Strengths, weaknesses, and development of Traditional Chinese Medicine in the health system of Hong Kong: through the eyes of future Western doctors. Journal of alternative and complementary medicine (New York, NY) 2006;12:185-189; discussion 191-182. 
24 Takahashi N, Udagawa N, Tanaka S, Suda T: Generating murine osteoclasts from bone marrow. Methods in molecular medicine 2003;80:129-144.

25 Galibert L, Tometsko ME, Anderson DM, Cosman D, Dougall WC: The involvement of multiple tumor necrosis factor receptor (TNFR)-associated factors in the signaling mechanisms of receptor activator of NF-kappaB, a member of the TNFR superfamily. The Journal of biological chemistry 1998;273:34120-34127.

26 Kajiya H: Calcium signaling in osteoclast differentiation and bone resorption. Advances in experimental medicine and biology 2012;740:917-932.

27 Novack DV, Yin L, Hagen-Stapleton A, Schreiber RD, Goeddel DV, Ross FP, Teitelbaum SL: The IkappaB function of NF-kappaB2 p100 controls stimulated osteoclastogenesis. The Journal of experimental medicine 2003;198:771-781.

28 Nakashima T, Hayashi $M$, Takayanagi $H$ : New insights into osteoclastogenic signaling mechanisms. Trends in endocrinology and metabolism: TEM 2012;23:582-590.

29 Nakamura H, Hirata A, Tsuji T, Yamamoto T: Role of osteoclast extracellular signal-regulated kinase (ERK) in cell survival and maintenance of cell polarity. Journal of bone and mineral research : the official journal of the American Society for Bone and Mineral Research 2003;18:1198-1205.

30 He Y, Staser K, Rhodes SD, Liu Y, Wu X, Park SJ, Yuan J, Yang X, Li X, Jiang L, Chen S, Yang

FC: Erk1 positively regulates osteoclast differentiation and bone resorptive activity. PloS one 2011;6:e24780.

31 Takayanagi H, Kim S, Koga T, Nishina H, Isshiki M, Yoshida H, Saiura A, Isobe M, Yokochi T, Inoue J, Wagner EF, Mak TW, Kodama T, Taniguchi T: Induction and activation of the transcription factor NFATc1 (NFAT2) integrate RANKL signaling in terminal differentiation of osteoclasts. Developmental cell 2002;3:889-901.

32 Whitmarsh AJ: Regulation of gene transcription by mitogen-activated protein kinase signaling pathways. Biochimica et biophysica acta 2007;1773:1285-1298.

33 Wagner EF, Matsuo K: Signalling in osteoclasts and the role of Fos/AP1 proteins. Annals of the rheumatic diseases 2003;62 Suppl 2:ii83-85.

34 Yavropoulou MP, Yovos JG: Osteoclastogenesis--current knowledge and future perspectives. Journal of musculoskeletal \& neuronal interactions 2008;8:204-216. 
FIG. 1

A

A
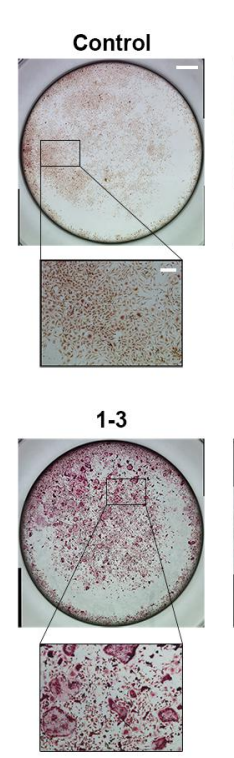

$$
\text { C }
$$
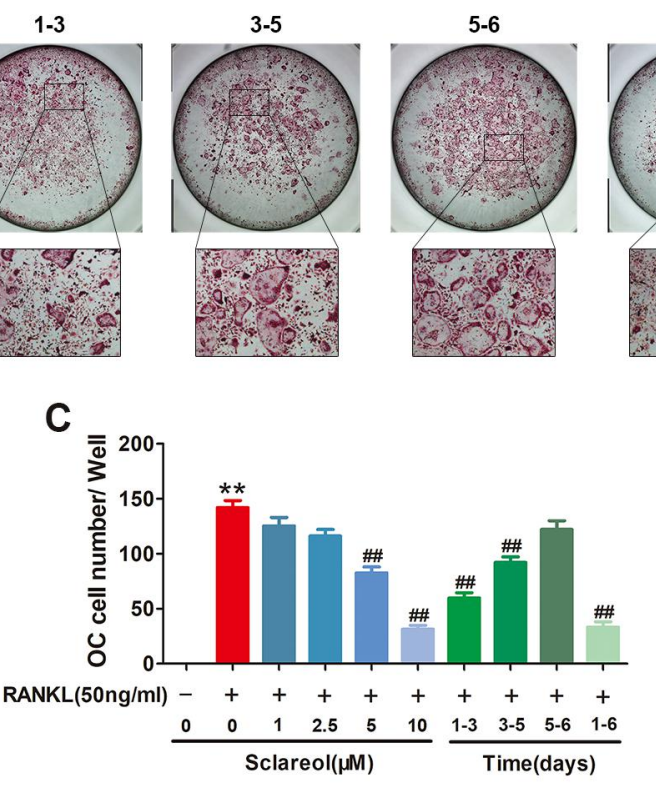

RANKL(50ng/mL)
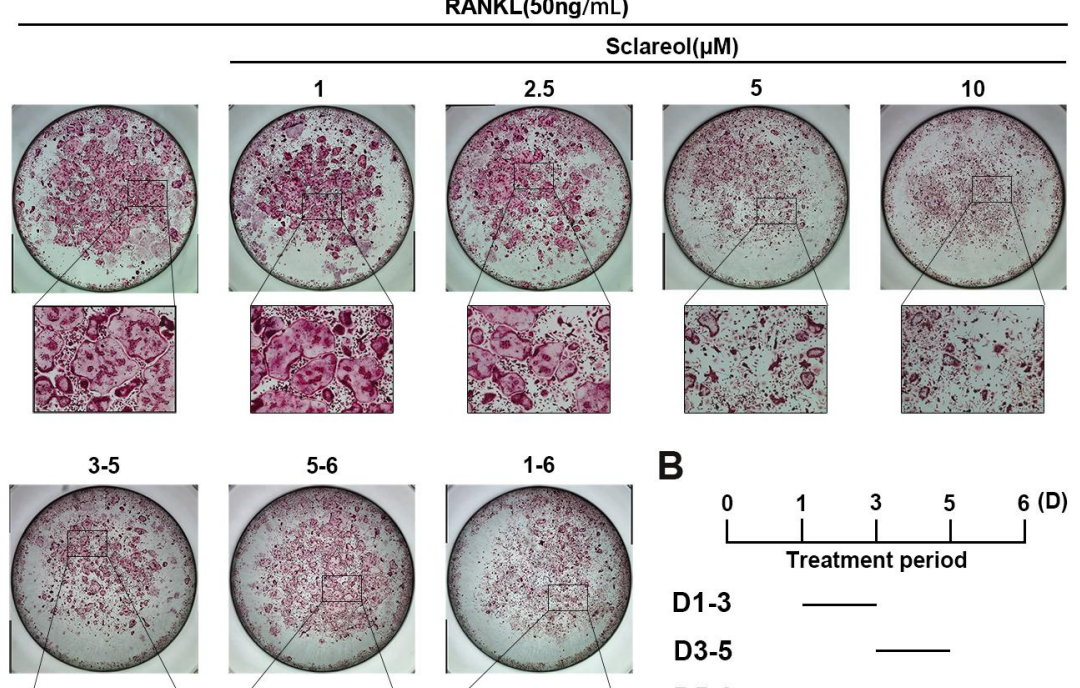

B

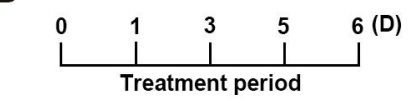

D1-3

D3-5

D5-6

D1-6

D

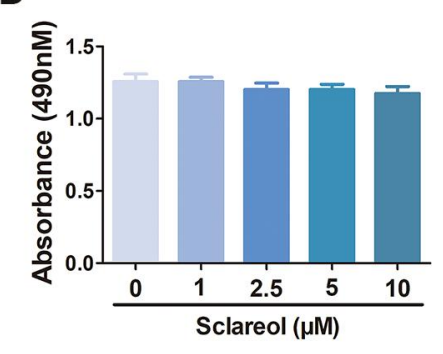


FIG. 2

A
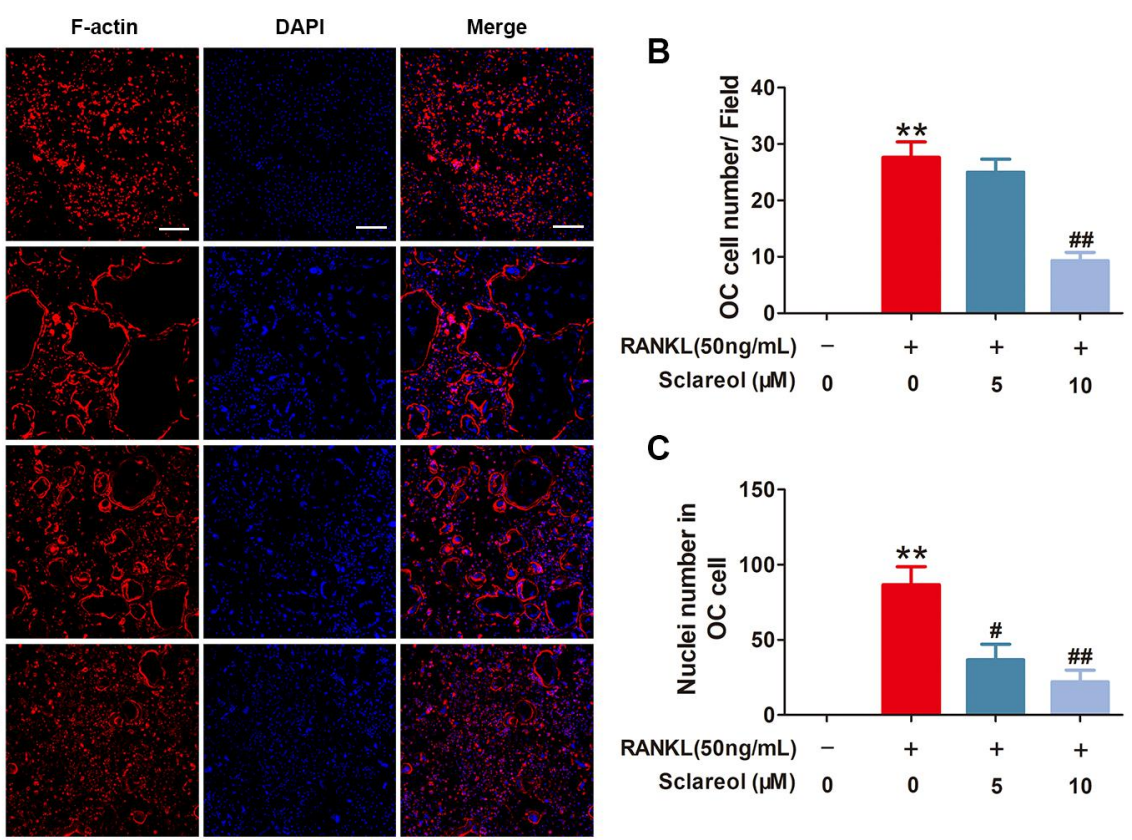

C

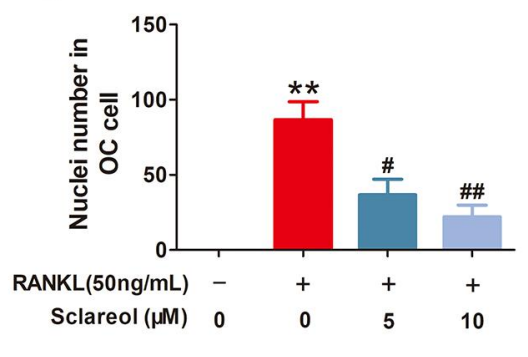


FIG. 3
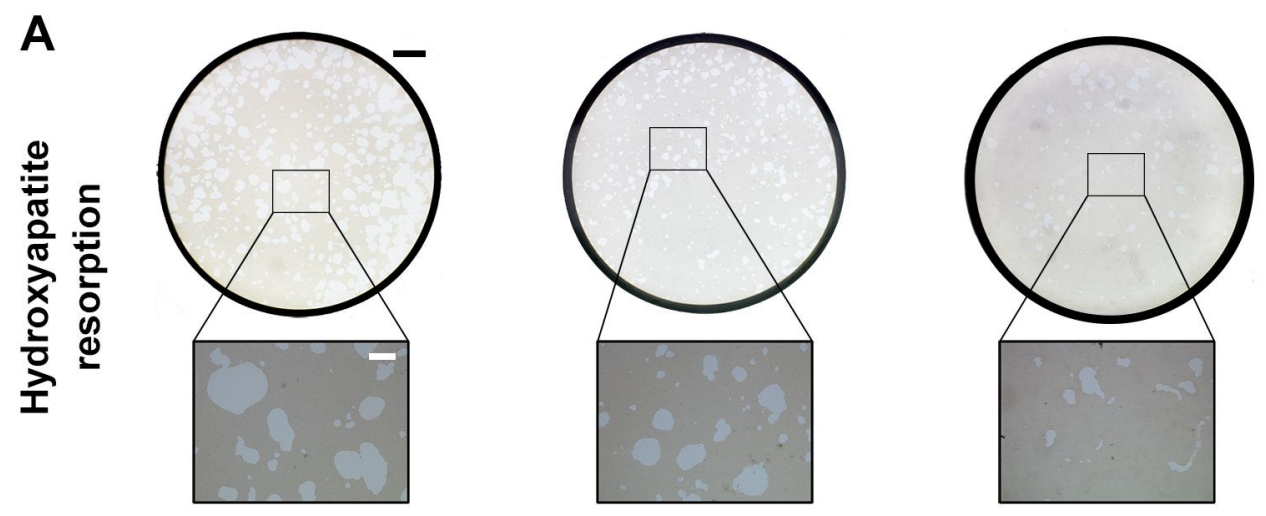

B

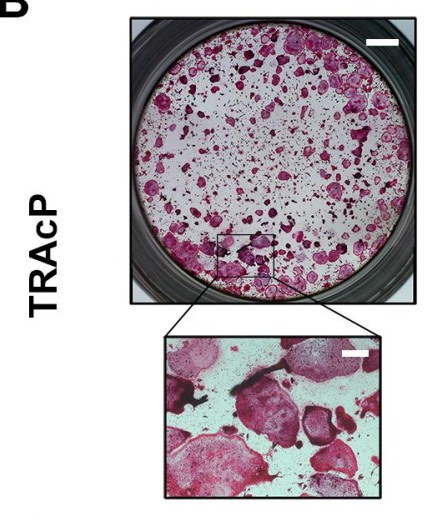

C
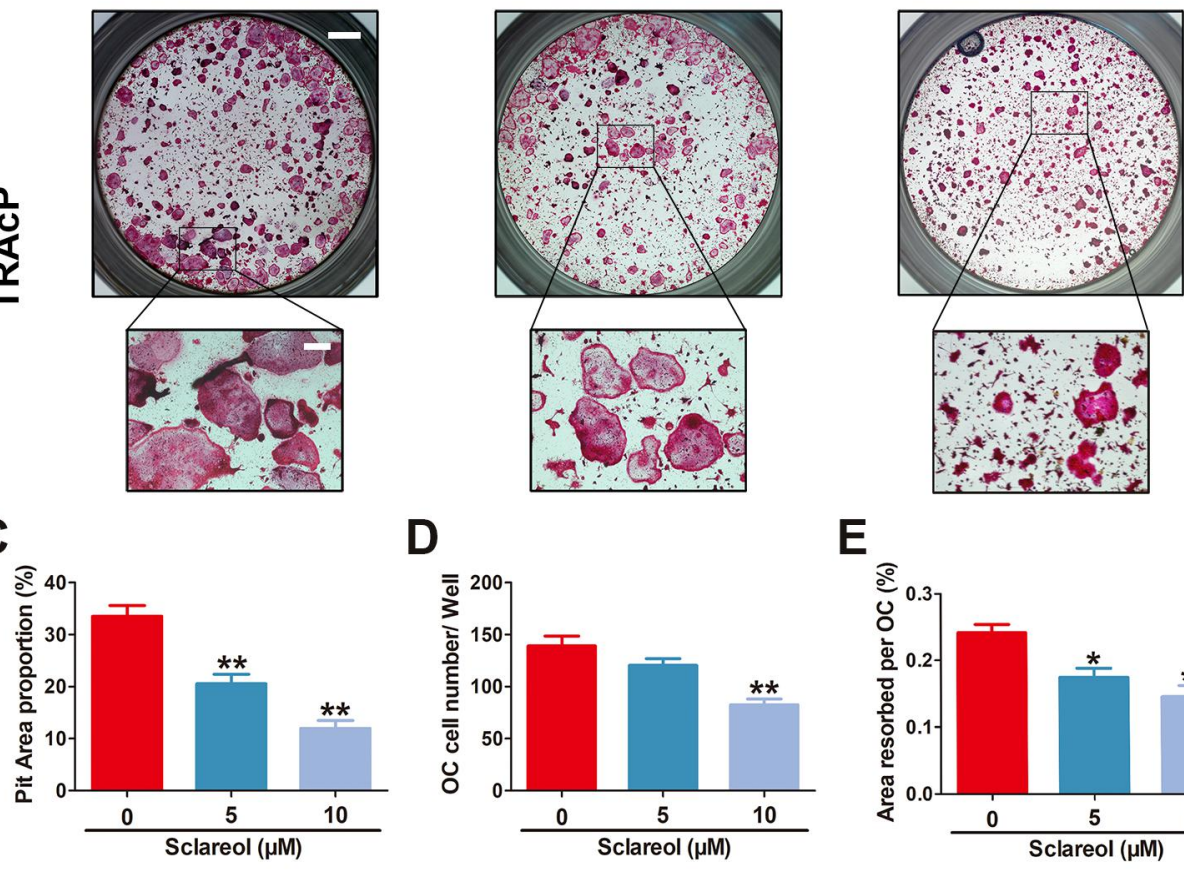

D

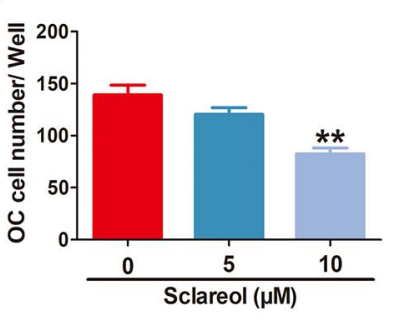

E

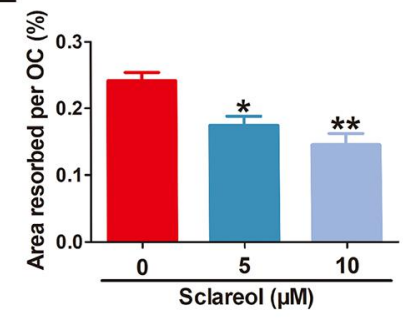


FIG. 4
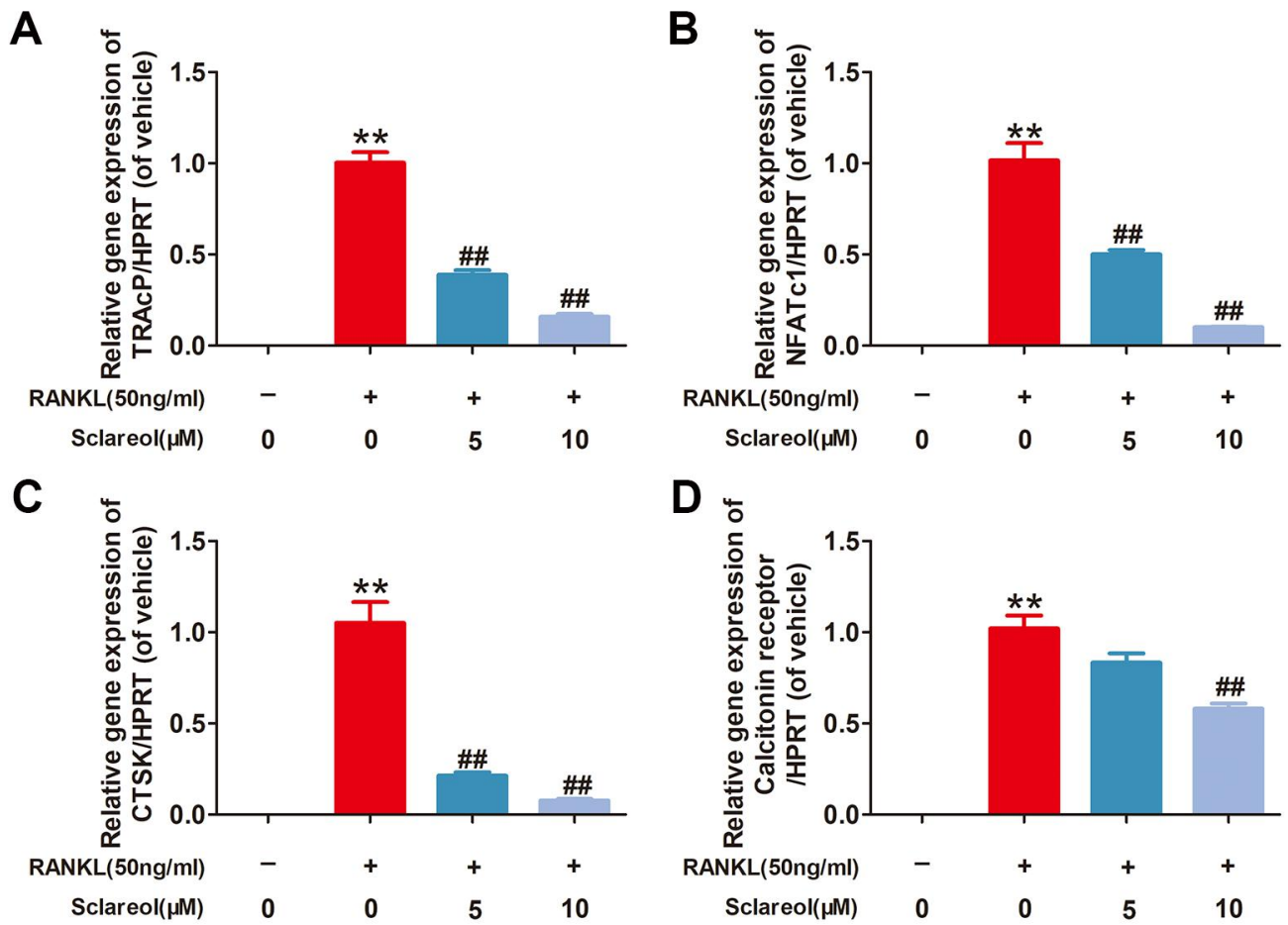

D ะ

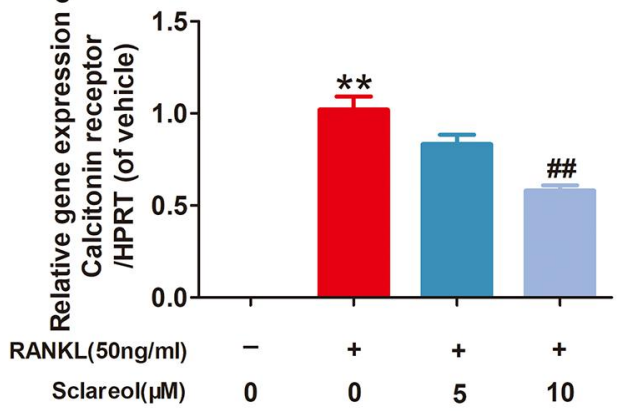


FIG. 5

A

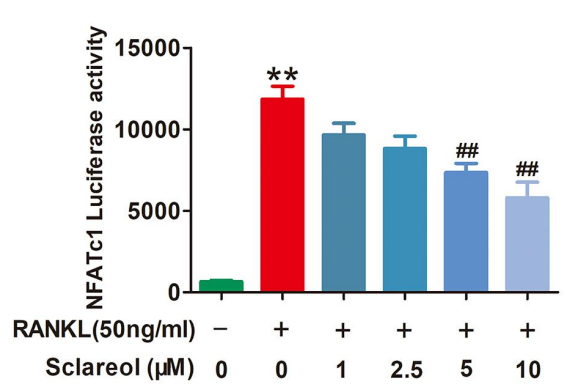

B

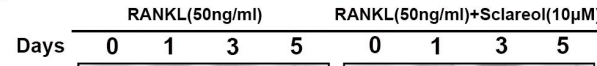

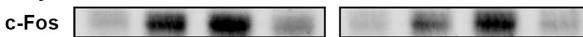

CTSK $\longrightarrow$

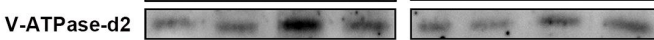

NFATc1 $\quad$ an

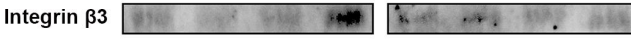

$\beta$-actin
C
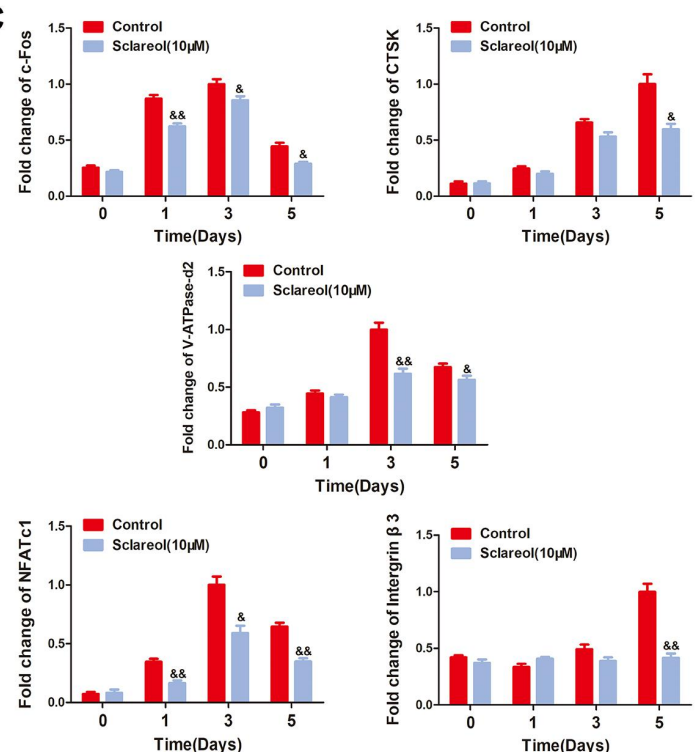

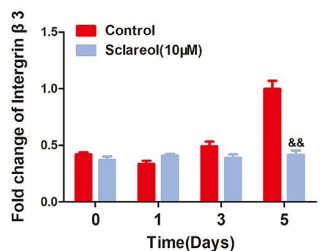


FIG. 6

A

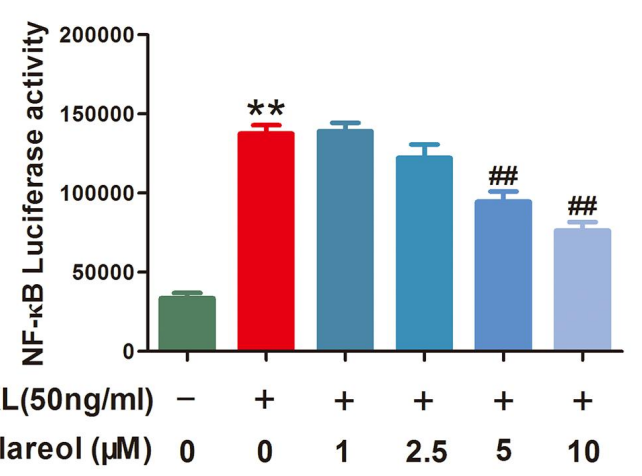

B
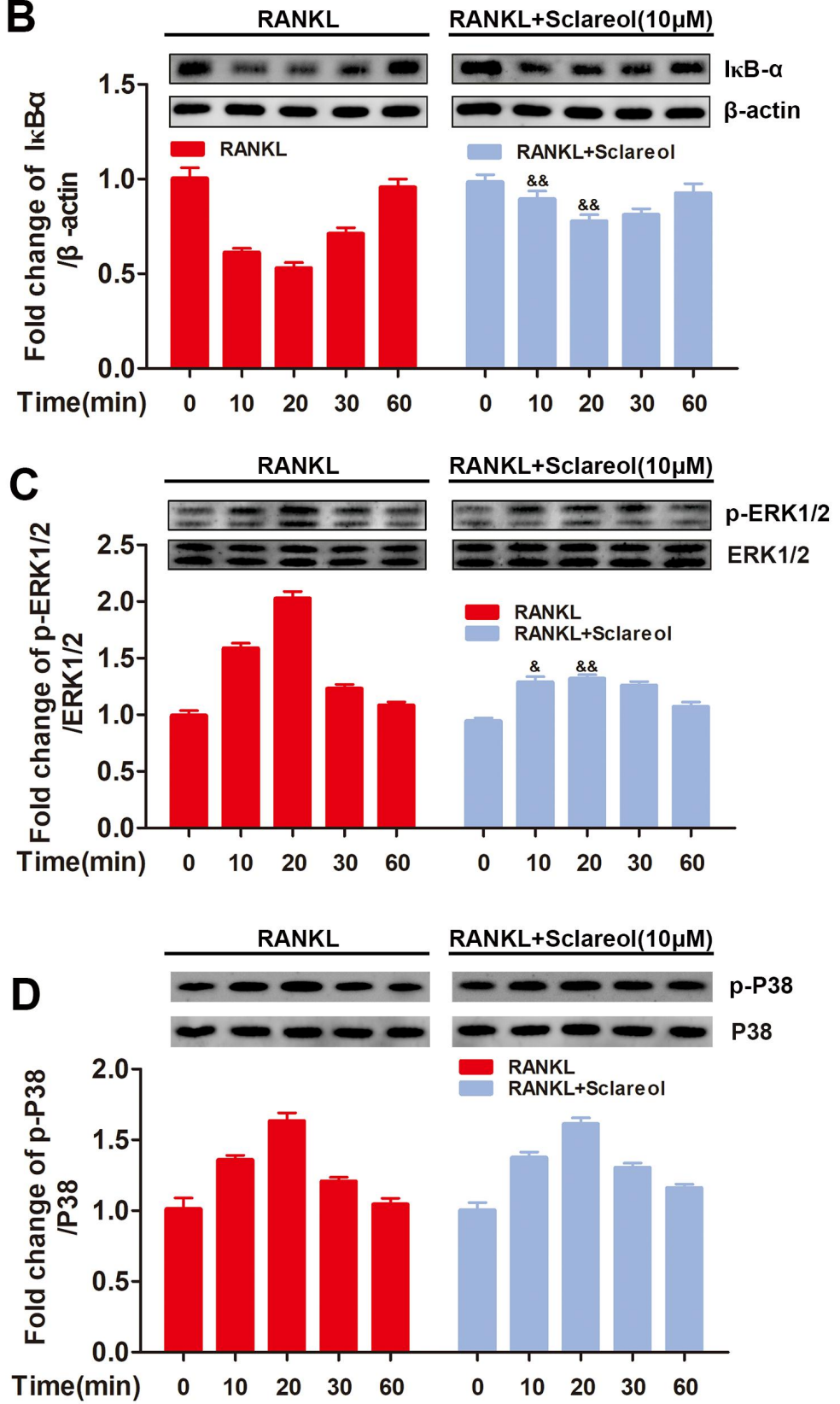
FIG. 7

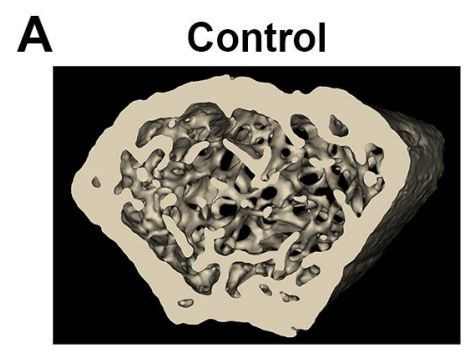

B
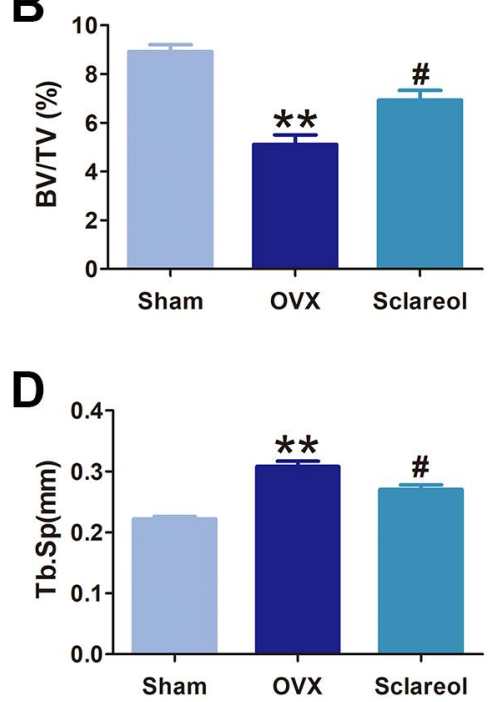

OVX

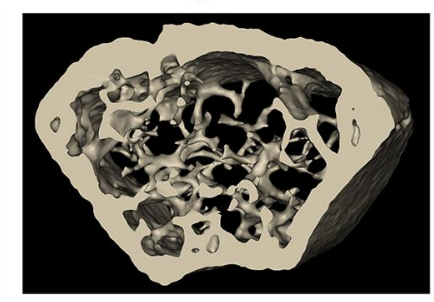

C

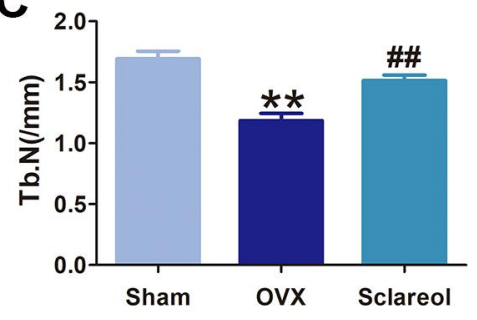

E

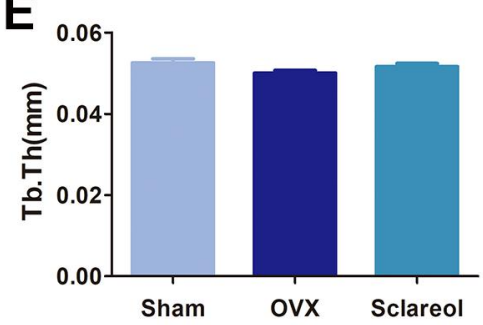


FIG. 8

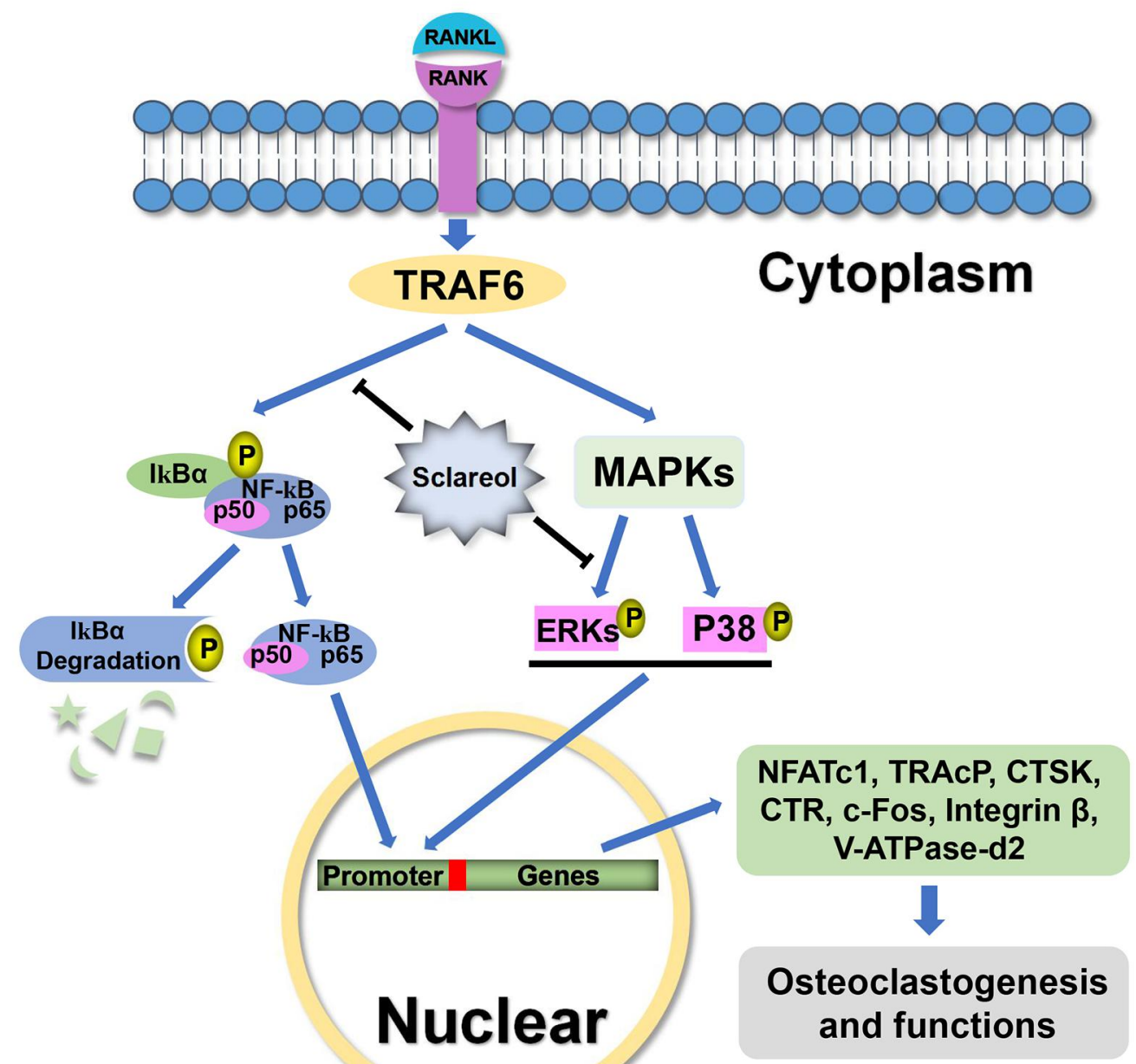


FIG. S1

A

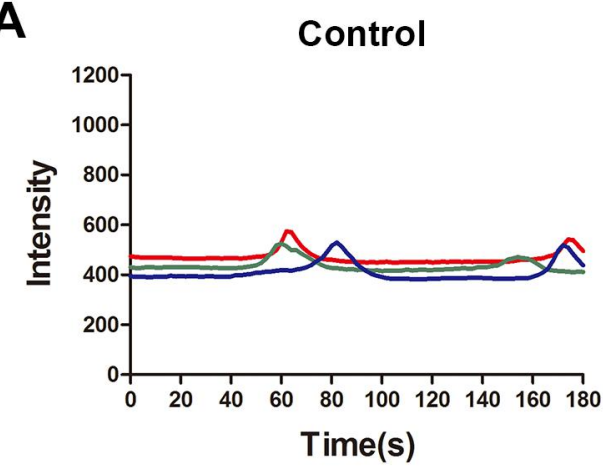

C

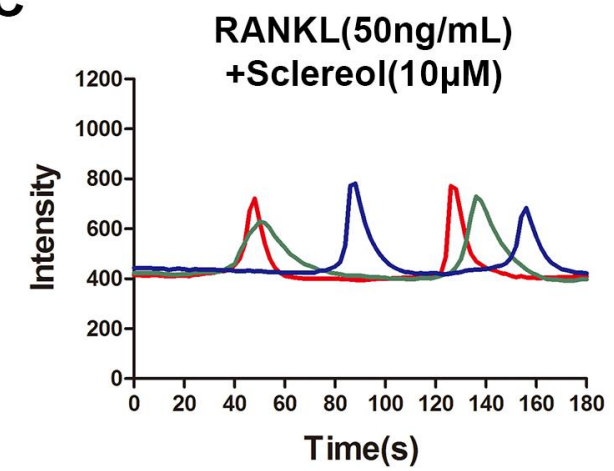

B

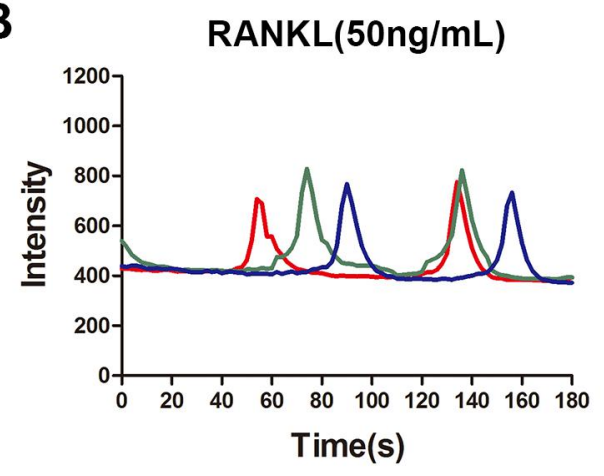

D

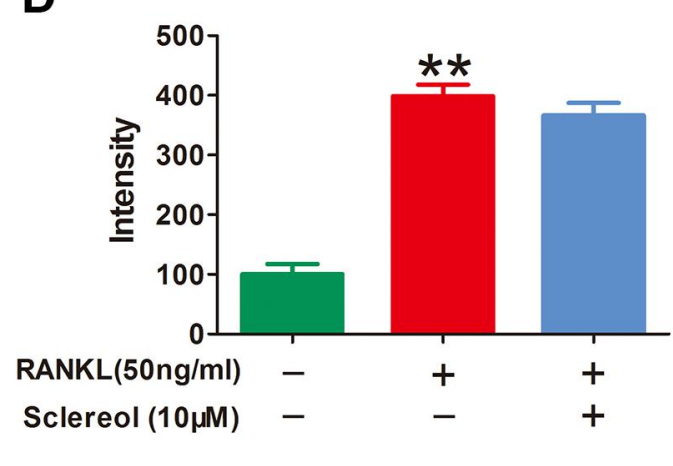

